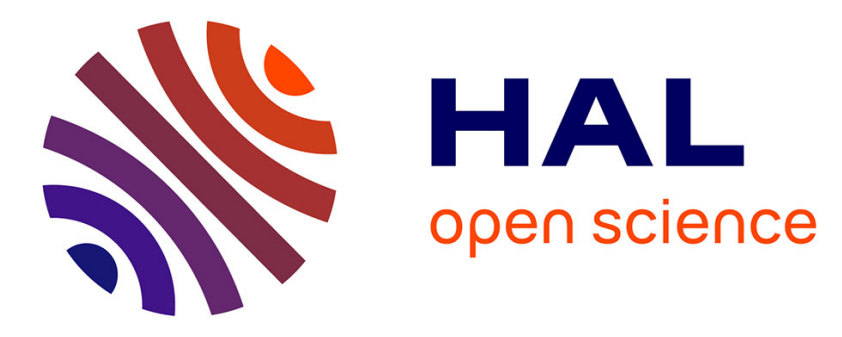

\title{
Dislocation density based crystal plasticity finite element model of polycrystals with grain boundary effect
}

\author{
Zhe Leng, Alankar Alankar, David Field, Nathalie Allain-Bonasso, Francis
}

Wagner

\section{- To cite this version:}

Zhe Leng, Alankar Alankar, David Field, Nathalie Allain-Bonasso, Francis Wagner. Dislocation density based crystal plasticity finite element model of polycrystals with grain boundary effect . 2nd World Congress on Integrated Computational Materials, May 2013, Salt Lake City, United States. pp.271-276. hal-01694522

\section{HAL Id: hal-01694522 \\ https://hal.science/hal-01694522}

Submitted on 27 Jan 2018

HAL is a multi-disciplinary open access archive for the deposit and dissemination of scientific research documents, whether they are published or not. The documents may come from teaching and research institutions in France or abroad, or from public or private research centers.
L'archive ouverte pluridisciplinaire HAL, est destinée au dépôt et à la diffusion de documents scientifiques de niveau recherche, publiés ou non, émanant des établissements d'enseignement et de recherche français ou étrangers, des laboratoires publics ou privés. 


\title{
DISLOCATION DENSITY BASED CRYSTAL PLASTICITY FINITE ELEMENT MODEL OF POLYCRYSTALS WITH GRAIN BOUNDARY EFFECT
}

\author{
Zhe Leng ${ }^{1}$, Alankar Alankar ${ }^{2}$, David P. Field ${ }^{1}$, Nathalie Allain-Bonasso ${ }^{3}{\text { Francis } \text { Wagner }^{3}}^{3}$ \\ ${ }^{1}$ School of Mechanical and Materials Engineering, Washington State University \\ ${ }^{2}$ Los Alamos National Laboratory, Los Alamos 87544, NM \\ ${ }^{3}$ LEM3, Univ. of Metz, Metz, France.
}

Keywords: Grain boundary, Geometrically necessary dislocation, Crystal plasticity, Finite element simulation

\begin{abstract}
:
Grain boundaries play an important role in determining the mechanical properties of metallic materials. The impedance of dislocation motion at the boundary results in a strengthening mechanism. In addition, dislocations can pile-up, be transmitted or be absorbed by the grain boundaries based on the local stress state and grain boundary character. In this study, a dislocation density based crystal plasticity finite element model is applied to incorporate the interaction between the dislocations and the grain boundaries, and a simulation is conducted on polycrystalline alpha iron deformed to $12 \%$ in uniaxial tension. The results indicate that the geometrically necessary dislocation density is generally higher near the grain boundary than within the grain interior. Taylor factor mismatch sometimes reveals strong localization effects near the grain boundaries.
\end{abstract}

\section{Introduction:}

Plastic deformation in metallic materials is controlled by dislocations. The dislocation movement on a slip plane and along a given slip direction under the influence of the local stress give rise to the permanent deformation, and the interaction between the dislocations results in forest hardening [1][2]. The dislocations also interact with the grain boundaries and can be absorbed, transmitted, or piled up at the boundaries, based on their character [3][4].

In order to investigate the effects of grain boundaries in metallic materials and the relationship between the microstructure and the mechanical properties, a uniaxial tension test has been applied to a polycrystalline alpha iron specimen, and the corresponding crystal plasticity finite element modeling is conducted based on the microstructure of the tested material.

\section{Crystal Kinematics:}

The kinematics of crystal plasticity formulation is based on the developments of Asaro and Rice [5], where the total deformation gradient $F$ is decomposed into an elastic component $F^{\theta}$ and a plastic component $F^{p}$ :

$F=F^{\theta} F^{p}$.

The plastic deformation gradient is the part due to slip, it corresponds to a stress free intermediate configuration, where the crystal lattice is undeformed and unrotated. The elastic 
deformation gradient involves the stretching and rotation of the crystal lattice.

The rate of plastic deformation gradient is given as:

$F^{p}=\left\{\sum_{\alpha=1}^{n} \dot{\gamma}^{\alpha} m_{0}^{\alpha} \otimes n_{0}^{\alpha}\right\} F^{p}$

$m^{\alpha}$ and $n^{\alpha}$ are the slip direction and slip plane normal of slip system $\alpha$ respectively. $m^{\alpha} \otimes n^{\alpha}$ is known as the Schmid tensor. In the current study, only $12<111>(110)$ slip systems are considered, and compared with the 24 and 48 slip system, it is more cost saving and follows the full model prediction in spite of a relatively higher local error.

\section{Constituitive Law:}

For a single crystal, the second Piola-Kirchoff stress is defined in the intermediate configuration: $\tau=\operatorname{det}\left(F^{\theta}\right) F^{\theta-1} \sigma F^{\theta-T}$

$\sigma$ is the Cauchy stress, the Piola-Kirchhoff stress is related to the work conjugate elastic Green strain $E^{\theta}$ through:

$\tau=\mathcal{L}: E^{\theta} \quad E^{\theta}=\frac{1}{2}\left(F^{\theta T} F^{\theta}-I\right)$

\section{Dislocation Density Based Framework:}

The dislocation density based formulation proposed by Arsenlis and Parks [6][7] is adopted, and as is briefly stated here. The dislocation evolves in the form of a square loop and each dislocation segment has pure edge or pure screw character with different polarities. The plastic strain rate from the Orowan equation is given as:

$\dot{\gamma}^{\alpha}=\left(\rho_{\theta+}^{\alpha} \bar{v}_{\theta+}^{\alpha}+\rho_{\theta-}^{\alpha} \bar{v}_{\theta-}^{\alpha}+\rho_{s+}^{\alpha} \bar{v}_{s+}^{\alpha}+\rho_{s-}^{\alpha} \bar{v}_{s-}^{\alpha}\right)\left|b^{\alpha}\right|$

where $b$ is the Burgers vector and $\bar{v}$ is the average velocity of the dislocation.

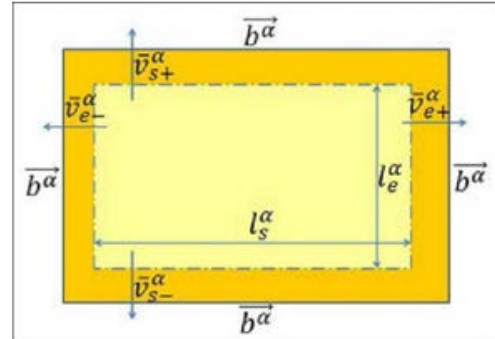

Fig.1 Illustration of the square dislocation loop assumed in the model framework. [7]

The evolution of dislocation includes generation, annihilation and dislocation flux terms:

$\dot{\rho}^{\alpha}=\dot{\rho}_{g e n}^{\alpha}+\dot{\rho}_{a n n}^{\alpha}+\dot{\rho}_{\text {flux }}^{\alpha}$

It is assumed that the generation of dislocation density mainly results from the expansion of the dislocation loop as shown in Fig.1, so the dislocation generation rate depends on the current dislocation state, such as density, velocity and average segment length.

$\dot{\rho}_{\mathrm{e}+(\text { gen })}^{\alpha}=\dot{\rho}_{\mathrm{e}-(\mathrm{gen})}^{\alpha}=\frac{\rho_{s+}^{\alpha}\left|\overline{\mathbf{v}}_{s+}^{\alpha}\right|}{\Gamma_{s+}^{\alpha}}+\frac{\rho_{s-}^{\alpha}\left|\overline{\mathbf{v}}_{s-}^{\alpha}\right|}{\Gamma_{s-}^{\alpha}}$ 
$\dot{\rho}_{g+\text { (gen) }}^{\alpha}=\dot{\rho}_{s-\text { (gen) }}^{\alpha}=\frac{\rho_{e+}^{\alpha}\left|\bar{v}_{e+}^{\alpha}\right|}{\bar{\Gamma}_{e+}^{\alpha}}+\frac{\rho_{e-}^{\alpha}\left|\bar{v}_{e-}^{\alpha}\right|}{\bar{l}_{e-}^{\alpha}}$

Dislocation annihilation results from the dipole interactions:

$\dot{\rho}_{e+(a n n)}^{\alpha}=\dot{\rho}_{\theta-(a n n)}^{\alpha}=-\rho_{\theta+}^{\alpha} \rho_{\theta-}^{\alpha} R_{\theta}\left(\left|\bar{v}_{\theta+}^{\alpha}\right|+\left|\bar{v}_{\theta-}^{\alpha}\right|\right)$

$\dot{\rho}_{\mathrm{s}+(\mathrm{ann})}^{\alpha}=\dot{\rho}_{\mathrm{s}-(\mathrm{ann})}^{\alpha}=-\rho_{\mathrm{s}+}^{\alpha} \rho_{\mathrm{s}-}^{\alpha} \mathrm{R}_{\mathrm{s}}\left(\left|\overline{\mathbf{v}}_{\mathrm{s}+}^{\alpha}\right|+\left|\overline{\mathrm{v}}_{\mathrm{s}-}^{\alpha}\right|\right)$

$R_{e}$ and $R_{g}$ are the critical radii of interactions for edge and screw dislocations, respectively.

In a discrete dislocation basis, the dislocation tensor is calculated as:

$\mathbf{A}=\sum_{\alpha}\left(\rho_{\mathrm{e}+}^{\alpha}-\rho_{\mathrm{e}-}^{\alpha}\right) \mathbf{b}_{0}^{\alpha} \otimes \mathbf{P}_{0}^{\alpha}+\left(\rho_{\mathrm{s}+}^{\alpha}-\rho_{\mathrm{s}-}^{\alpha}\right) \mathbf{b}_{0}^{\alpha} \otimes \mathrm{m}_{0}^{\alpha}$

and it is related to plastic deformation by:

$\mathbf{A}=\operatorname{Curl}\left(\mathbf{F}^{p}\right) \mathbf{F}^{\mathrm{p}^{\mathrm{T}}}$

by taking the time rate form of the above two equations and making some physical

considerations, for example, accumulation of a certain amount of $\rho_{\mathrm{e}+}^{\alpha}$ is equal to loss of the same amount of $\rho_{\mathrm{e}-}^{\alpha}$, the dislocation flux divergence of each type of dislocation can be calculated [7]. In the current study, it is assumed that the GND is only a subset of total dislocation density, and the GND density of a specified type of dislocation can be quantified by the net polarity of that type of dislocation. In other words, both the dislocation flux and the GND density mainly depend on the local plastic deformation history.

Dislocation resistance is mainly controlled by forest dislocations, and is represented in a Taylor type equation of hardening:

$S_{d}^{\alpha}=\mu b \sqrt{\sum_{\beta=1}^{N}\left(G^{\alpha \beta} \rho^{\beta}\right)}$

where $G$ is the dislocation strength interaction matrix. Dislocation velocity $\mathrm{v}^{\alpha}$ is calculated as:

$\bar{v}_{e}^{\alpha}=v_{e} \exp \left\{-\frac{F_{e}}{k T}\left(1-\left(\frac{\left|\tau^{\alpha}\right|}{S_{d e}}\right)^{p e}\right)^{q e}\right\}$

$\bar{v}_{s}^{\alpha}=\frac{L b^{2}}{1_{c}^{2}} v_{d} \exp \left\{-\frac{F_{s}}{k T}\left(1-\left(\frac{\left|\tau^{\alpha}\right|}{\xi}\right)^{p s}\right)^{q a}\right\}$

where $\mathrm{T}$ is the absolute temperature, $\mathrm{k}$ is Boltzmann's constant, $\mathrm{L}$ is segment length, $\mathrm{I}_{\mathrm{c}}$ is the critical length for double kink nucleation, $v_{d}$ is the Debye frequency. $\xi$ is the Peierls stress on screw dislocations. $\tau^{\alpha}$ is the Schmid stress. It should emphasized here that although the Schmid rule is often a good approximation for simple metals, the violation of Schmid law in BCC metals is inevitable.[8].

\section{Grain Boundary Effect:}

The grain boundaries act as obstacles for dislocation motion. When they encounter a grain boundary, mobile dislocations will accumulate at the grain boundary in the form of pile ups and give rise to the stress concentration there. Among all the types of interactions between dislocations and grain boundaries, slip transmission makes important contribution to the polycrystalline deformation, and the grain boundary effect is included in the model by introducing an thermally activated slip transmission concept [9]. The activation enthalpy quantifies the transmission probability for the mobile dislocations and it is considered as an energy barrier for those grain boundary elements. This energy barrier $E_{G B}^{a}$ is equal to the elastic 
energy of forming the misfit dislocation at the interface [9][10].

$l^{\alpha^{\prime}}=l^{\alpha}\left(\boldsymbol{n}_{g b} \times \boldsymbol{n}^{\alpha}\right)$

$\boldsymbol{l}^{\beta^{\prime}}=l^{\beta}\left(\boldsymbol{n}_{g b} \times \boldsymbol{n}^{\beta}\right)$

$E_{G B}^{\alpha}=\frac{1}{2} *\left(l_{G B}^{\alpha} G b_{G B}^{\alpha}{ }^{2}\right)$

$\boldsymbol{l}^{\alpha \prime}$ is incoming dislocation segment, and $\boldsymbol{l}^{\beta^{\prime}}$ is outgoing dislocation segment,:

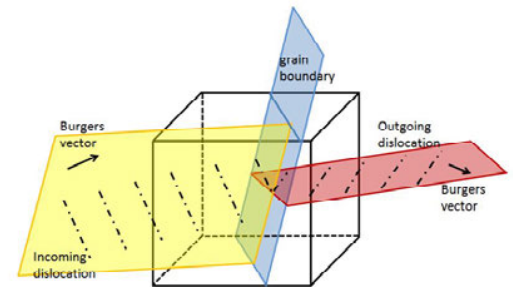

Fig.2 Illustration of slip transmission behavior.[9]

It is likely that the slip transmission will occur at the minimum energy expenses, thus for the grain boundary elements, for each incoming slip system, all the possible outgoing slip system will be investigated, the one with minimum energy barrier will be considered as the corresponding outgoing slip system.

$E_{G B}^{\alpha}=\frac{1}{2} \min _{\beta} G b_{G B}^{\alpha}{ }^{2} l_{G B}^{\alpha}$

The grain boundaries in the model are represented by the bi-crystal volume elements [11], each having the crystallographic lattice orientations of its adjacent crystals. The grain boundary effects are considered on the near boundary bi-crystal volume elements. The $F_{\text {eff }}^{\alpha}$ will be used instead of $\mathrm{F}_{\mathrm{e}}$ or $\mathrm{F}_{\mathrm{s}}$ compared with the ingrain elements.

$F_{\text {eff }}^{\alpha}=\mathrm{F}_{\mathrm{e} / \mathrm{s}}+E_{G B}^{\alpha}$

\section{Uniaxial Tensile Test:}

The uniaxial tension test was conducted on polycrystalline ferrite. The material was subjected to $12 \%$ strain, and the simulation was conducted only on a small region with about 6 grains. The crystal orientation map of the small region was taken before and after the experiment in order to investigate the microstructure evolution.
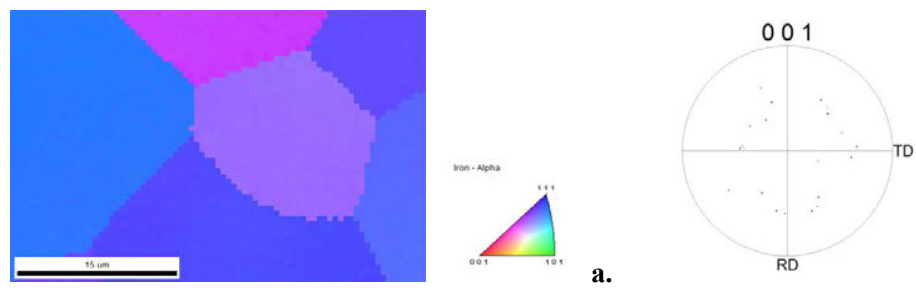

Fig.3 a) Orientation image map and b) Pole figure of alpha-iron before deformation. 


\section{Simulation Results and Concusions:}

The effective stress and the statistical dislocation density distribution is shown in the following Fig.4a and Fig.4b, respectively. Statistical dislocation density is another subset of the total dislocation density and it is about the same as the total dislocation density, since most of the dislocation in the total dislocation density do not contribute to geometric effect such as lattice curvature. A high stress state is likely to be located at grain boundaries. As a result of the stress concentration at these boundaries, a high dislocation density state is more favored there.
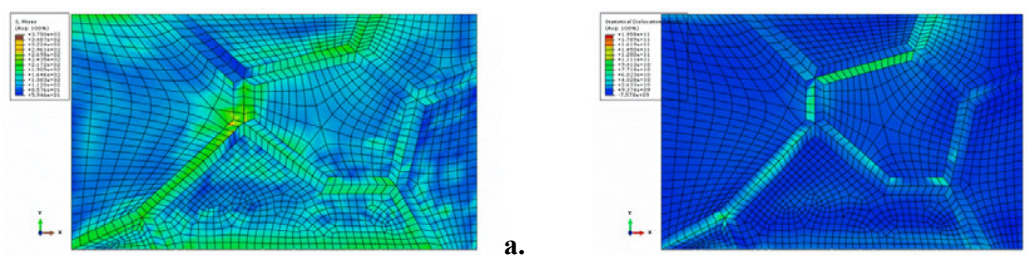

Fig.4 The simulation result of a) Effective stress and b) statistical dislocation distribution of alpha-iron after tension

Fig.5a is micromechanical Taylor factor [12] determined from the simulation, it is calculated from the local stress and strain state. Most of the region has a Taylor factor between 1 to 4 , and the Taylor factor near the grain boundaries is higher than the grain interior, indicating a higher plastic effect near these regions.

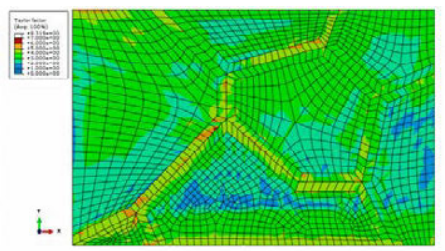

a.

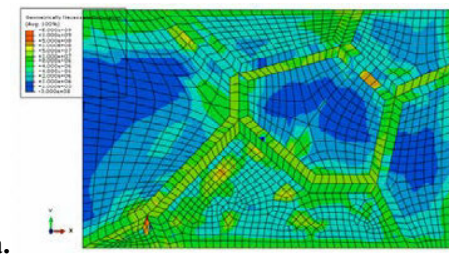

b.

Fig.5 a) Taylor factor and b) Geometrically necessary dislocation determined from the model

b.

One

important role of geometrically necessary dislocations is to maintain the lattice curvature [13][14], thus the geometrically necessary dislocation here is derived from the plastic strain gradient [15]. Fig.5b is the GND density determined from the model, it is about $1 \%$ of the total dislocation density, and higher GND density is localized near the grain boundaries and triple junctions.

Fig.6b is the predicted texture of the polycrystal after deformation, compared with the experimental result in Fig.6a. The results are similar, and both of them indicate orientation spread during deformation. 

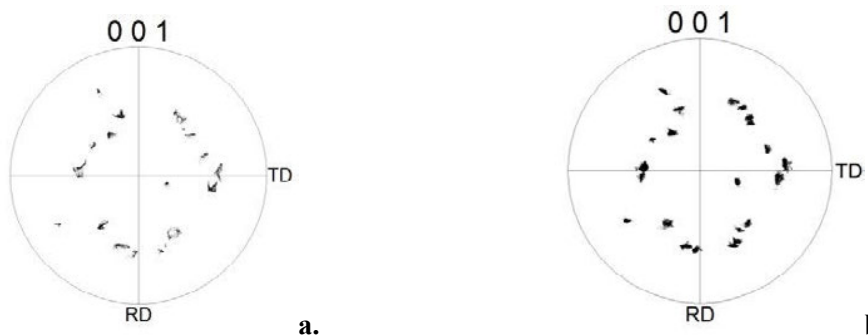

Fig. 6 The 001 pole figure from a) experimental result and b) simulation result.

The simulation shows the stress localization and increased dislocation concentration at grain boundaries, and the high Taylor factor at the interfaces and triple junctions indicates the higher hardness there. The texture prediction is acceptable in this model.

\section{Reference:}

[1] M. Tang, M. Fivel, L. P. Kubin, Materials Science and Engineering A309-310 (2001) 256260.

[2] B. Devincre, L. Kubin, T. Hoc, Scripta Materialia 54 (2006) 741-746.

[3] A. Acharya, Philosophical Magazine Vol. 87, Nos. 8-9, 11-21 March 2007, 1349-1359.

[4] H. Lim, M.G. Lee, J.H. Kim, B.L. Adam, R.H. Wagoner, International Journal of Plasticity 27 (2011) 1328-1354.

[5] Asaro, R.J. and Rice, J.R., J.Mech.Phys. Solids, 25, 309-338, 1977.

[6] Athanasios Arsenlis, David M. Parks, Richard Becker, Vasily V. Bulatov, Journal of the Mechanics and Physics of Solids, 52 (2004) 1213 - 1246.

[7] Athanasios Arsenlis, David M. Parks, Journal of the Mechanics and Physics of Solids, 50 (2002) 1979 - 2009.

[8] Duesbery. M.S., Vitek, V. and Cserti, J. :Non-Schmid plastic behaviour in bcc metals and alloys in Understanding Materials: A Festschrift for Sir Peter Hirsch, edited by C. J. Humphreys, The Institute of Materials, London, p. 165, 2002.

[9] A. Ma, F. Roters, D. Raabe, Acta Materialia 54 (2006) 2181-2194.

[10] A. Ma, F. Roters, D. Raabe, International Journal of Solids and Structures 43 (2006) 72877303.

[11] L.P. Evers, D.M. Parks, W.A.M. Brekelmans, M.G.D. Geers, Journal of the Mechanics and Physics of Solids, 50 (2002) $2403-2424$.

[12] D. Raabe, M. Sachtleber, Z. Zhao, F. Roters and S. Zaefferer, Acta mater. 49 (2001) 34333441.

[13] M. F. Ashby, (1970) Philosophical Magazine, 21: 170, 399 — 424.

[14] J. F. Nye, (1953) Acta Metallurgica, 1, 153-162.

[15] Tetsuya Ohashi, Philosophical Magazine Letters, 1997, Vol. 75, NO. 2, 51- 57. 